\title{
Merging Ayurvedic Ashwagandha with Traditional Chinese Medicine Part 1. Foundation in Ashwagandha: Physiological Effects, Clinical Efficacy, and Properties
}

\author{
Michael Forman ${ }^{1,2}$ and Nicholas A. Kerna ${ }^{1 *}$ \\ ${ }^{1}$ College of Medicine, University of Science, Arts and Technology, Montserrat, BWI \\ ${ }^{2}$ Atlantic Institute of Oriental Medicine, USA
}

"Corresponding author: Nicholas A. Kerna, College of Medicine, University of Science, Arts and Technology, 4288 Young field Street, Wheat Ridge, CO 80033 USA. Email: nicholas.kerna@usat.edu

Citation: Forman M, Kerna NA (2018) Merging Ayurvedic Ashwagandha with Traditional Chinese Medicine Part 1. Foundation in Ashwagandha: Physiological Effects, Clinical Efficacy, and Properties. Curr Res Complement Altern Med. CRCAM-133. DOI:10.29011/ 2577-2201/100033

Received Date: 08 September, 2018; Accepted Date: 04 October, 2018; Published Date: 12 October, 2018

\begin{abstract}
Traditional Chinese Medicine (TCM) has a methodical and organized system for the classification of herbal medicines. These classifications have been developed over many hundreds of years of careful observation and documentation of each of the traditional herbs and their effect on the human body. Due to isolation and long distances between different countries and cultures, certain significant herbal medicines (such as Ashwagandha from India) are not currently classified per TCM principles.

The systems of Ayurveda and TCM are juxtaposed, herein, to establish a parallel between the two systems. In TCM, each herbal substance has its distinct attributes; therefore, a side-by-side comparison was made between each relevant herb and Ashwagandha. Because of this comparison, in the TCM herbal classification system, Ashwagandha can be categorized as "Tonify Qi" and "Tonify Blood and Essence". This comparison, depicted in (Table 1), provides evidence to classify Ashwagandha in TCM accurately and establishes a methodology by which other relevant herbal medicines can be joined with Chinese medicine and classified under TCM categories and terms.
\end{abstract}

Keywords: Abortifacient; Adaptogen; Ashwagandha; Ayurveda; Chínese Medicine; Dunal; Qi; Materia Medica; TCM; Withania somnifera

\section{Abbreviations}

PDR : $\quad$ Physicians' Desk Reference

TCM : $\quad$ Traditional Chinese Medicine

\section{Preface}

The Compendium of Materia Medica (written by Li Shizhen), upon which TCM is based, lists and classifies, among grasses and plants, those herbs that were found or known to exist in China at the time of its writing during the Ming Dynasty (the first draft completed in 1578). The essence and purpose of this paper is to propose well-documented herbs outside of the historical
TCM "universe" be included in Materia Medica; and by doing so, certain herbal medicines, such as Ashwagandha (Withania somnifera) -a foundational herb in traditional Indian Ayurveda (Ayurvedic Medicine)-can be used effectively in accordance with existing TCM diagnostic criteria and treatment protocol.

Withania somnifera and Withania somnifera (Dunal) are interchangeable scientific names for the herb Ashwagandha. Michel Felix Dunal (1789-1856) was a French botanist and professor of botany in Montpellier, France. Some herbal experts use "Dunal"to acknowledge the professor involved in classifying the Solanaceous plant genus, under which Ashwagandha is listed. (The name Withernia somnifera is sometimes seen in the literature, mistakenly or not.)

This paper is the first in a series of papers; the series title being "Merging Ayurvedic Ashwagandha with Traditional 


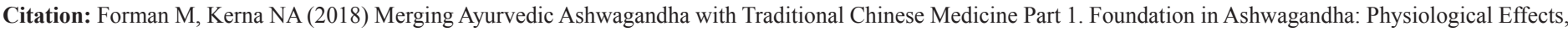
Clinical Efficacy, and Properties. Curr Res Complement Altern Med. CRCAM-133. DOI:10.29011/ 2577-2201 /100033

Chinese Medicine". This first paper's subtitle is "Foundation in Ashwagandha: Physiological Effects, Clinical Efficacy, and Properties". The subsequent papers will investigate apoptogenic herbs; physiological effects; TCM herb classification and Ayurvedic herb classification, and a comparison thereof; and a conclusion that supports the recognition of Ashwagandha in TCM and its inclusion in Materia Medica which is TCM's herbal equivalent to Western medicine's pharmaceutical PDR. Each paper is standalone, and can be read as such if the reader simply has an interest in one topic. However, the papers will also be linked resulting in an in-depth review of the herb, Withania somnifera (Ashwagandha), and its proposed inclusion and role as a fundamental herb in TCM.

\section{Introduction}

\section{The Proposition for the Inclusion of Ashwagandha in TCM}

Traditional Chinese Medicine (TCM) has a 5,000-year history and describes the 2,000-year-old tradition of medical theories and herbal classification and applications for the intervention in and prevention of illness and for maintaining health and well-being. In TCM, the primary therapeutic component is herbal substances. The herbs utilized have been methodically classified and organized following Chinese medical theories, which have culminated in several monumental works detailing the discovery and therapeutic development of herbal medicines. The following are three classical written works (Chinese herbal texts) dating back to $125 \mathrm{CE}$ :

- Shen Nong's Herbal Classic (Shen Nong Ben Cao Jing) (200$250 \mathrm{CE})$

- $\quad$ Materia Medica Tang Edition (Tang Ben Cao) (1057 CE)

- Compendium of Materia Medica (Bencao Gangmu) (1578 $\mathrm{CE})$

- Two contemporary texts in English are as follows:

- Chinese Medical Herbology and Pharmacology, 1st Edition, by J. Chen and T. Chen (2004)

- $\quad$ Materia Medica, 3rd Edition, by Dan Bensky, et al. (2015)

In the past century, with the worldwide dominance of Western medical science and its pharmaceutical intervention orientation to health management, many herbs have been counterclassified regarding their physiological effects and potential. These herbs have now been arranged based on their direct physiological impact on the body from a microbiological perspective. In this regard, many herbs fail to meet scientific criteria on par with the anecdotal evidence of historical and contemporary proponents and users of the herbs or derivatives. Empirical studies are required to prove efficacy. These studies and observations must be of high quality-with peer-review acceptance-making them suitable for the scientific and medical communities.

The TCM "herbal classics" include mainly herbs found in China and surrounding areas; those were the herbs principally at hand during the period these herbs were commonly used, and subsequently documented. However, some of the herbs included were those found in other parts of the world, beyond China, where they were recognized for medicinal properties in those different cultures and countries. As trade foreign trade found its way with China, "foreign" herbs found their way into the TCM system through the purview of Chinese herbal medicine scholars and authorities [1].

Withenia somnifera (Dunal) is referred to in Indian Ayurvedic medicine as Ashwagandha. This extraordinary herb, with its significant healing and medicinal properties, warrants inclusion in the TCM Materia Medica. In this way, Ashwagandha would be included in TCM textbooks, taught at TCM schools, and recognized by TCM practitioners as an effective herbal medicine in the treatment and prevention of certain diseases (Table 1).

\begin{tabular}{|c|l|l|c|l|l|l|}
\hline $\mid$ & Ren Shen & Huang Qi & $\begin{array}{c}\text { Ci Wu } \\
\text { Jia }\end{array}$ & Lu Rong & Dang Gui & Ashwagandha \\
\hline $\begin{array}{c}\text { Taste and } \\
\text { Temperature }\end{array}$ & $\begin{array}{l}\text { Sweet, } \\
\text { slightly } \\
\text { bitter, } \\
\text { slightly } \\
\text { warm }\end{array}$ & $\begin{array}{l}\text { Sweet, } \\
\text { slightly } \\
\text { warm }\end{array}$ & $\begin{array}{l}\text { Sweet, } \\
\text { neutral }\end{array}$ & $\begin{array}{l}\text { Sweet, } \\
\text { salty, } \\
\text { warm }\end{array}$ & $\begin{array}{l}\text { Sweet, } \\
\text { acrid, } \\
\text { warm }\end{array}$ & Sweet, acrid \\
\hline $\begin{array}{c}\text { Category } \\
\text { Classification }\end{array}$ & Tonify Qi & Tonify Qi & Tonify Qi & $\begin{array}{l}\text { Tonify } \\
\text { Yang } \\
\text { (Essence) }\end{array}$ & $\begin{array}{l}\text { Tonify } \\
\text { Blood }\end{array}$ & $\begin{array}{l}\text { Tonify } \\
\text { Defensive Qi }\end{array}$ \\
\hline $\begin{array}{c}\text { Channels } \\
\text { Entered }\end{array}$ & $\begin{array}{l}\text { Lung } \\
\text { Spleen }\end{array}$ & $\begin{array}{l}\text { Lung } \\
\text { Spleen }\end{array}$ & $\begin{array}{l}\text { Spleen } \\
\text { Kidney }\end{array}$ & $\begin{array}{l}\text { Kidney } \\
\text { Liver }\end{array}$ & $\begin{array}{l}\text { Heart } \\
\text { Liver } \\
\text { Spleen }\end{array}$ & $\begin{array}{l}\text { Kidney } \\
\text { Spleen } \\
\text { Lung }\end{array}$ \\
\hline $\begin{array}{c}\text { Superior } \\
\text { Herb }\end{array}$ & Yes & Yes & Yes & Yes & Yes & Yes \\
\hline $\begin{array}{c}\text { Adapto- } \\
\text { genic }\end{array}$ & Yes & Yes & Yes & Yes & Yes & Yes \\
\hline $\begin{array}{c}\text { Immuno- } \\
\text { modulating }\end{array}$ & Yes & Yes & Yes & Yes & Yes & Yes \\
\hline $\begin{array}{c}\text { Neuro- } \\
\text { protective }\end{array}$ & Yes & Yes & Yes & Yes & Yes & Yes \\
\hline $\begin{array}{c}\text { Hemato- } \\
\text { poietic }\end{array}$ & No & No & No & No & Yes & Yes \\
\hline $\begin{array}{c}\text { Anti- } \\
\text { inflammatory }\end{array}$ & Yes & Yes & Yes & Yes & Yes & Yes \\
\hline $\begin{array}{c}\text { Anti- } \\
\text { cancer }\end{array}$ & Yes & Yes & Yes & Yes & Yes & Yes \\
\hline
\end{tabular}

Table 1: Comparison of Ashwagandha and classic Chinese herbs.

Note: Comparison of characteristics of Ashwagandha with traditional TCM herbs evidencing Ashwagandha's similarities thus supporting the case for its inclusion in Materia Medica. Copyright (C) 2016 by Michael Forman.

\section{Classification and Etymology of Ashwagandha}

Ashwagandha is scientifically classified as Withania somnifera (Dunal); it belongs to the botanical family Solanaceae. Long revered as a cure-all herb in India and its importance in Ayurvedic medicine. Ayurveda is a traditional system of healing 


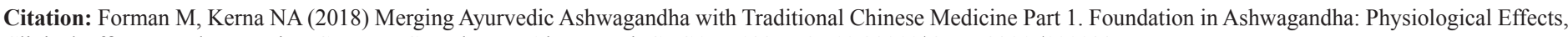
Clinical Efficacy, and Properties. Curr Res Complement Altern Med. CRCAM-133. DOI:10.29011/ 2577-2201 /100033

that can be traced back to 6,000 BCE. Ashwagandha's reputation is one of the essential herbs in Ayurvedic medicine. This "famous" herb is classified as Rasayana (a tonic), e.g., a group of plants having properties to rejuvenate the body, promote the body's defense against disease, slow the aging process, and enhance memory [2].

Ashwagandha derives its name from the Sanskrit words "ashva" (meaning horse) and "gandha" (meaning smell) due to the horse-like odor of the roots. Another reason for its name is the belief that consuming the plant's extracts will give a person the strength and vitality of a horse [3].

Customarily in India, the root of the plant is considered the crucial component which is crushed and used as a fine powder, called "churna". This powder form allows for easy blending with water, milk, honey or other fluids [4]. Ashwagandha has been used as an aphrodisiac, liver tonic, diuretic, astringent, and anti-inflammatory Withania Somnifera, 2004. It has been given to children as a tonic and to elderly for rheumatism, insomnia, constipation, and nervous breakdown [4].

\section{Description of Ashwagandha}

Ashwagandha grows as a small, woody shrub, usually 2 to 5 feet tall. It has oval, small yellowish-green leaves with five-petaled flowers and has red and yellow berries (Figure 1).

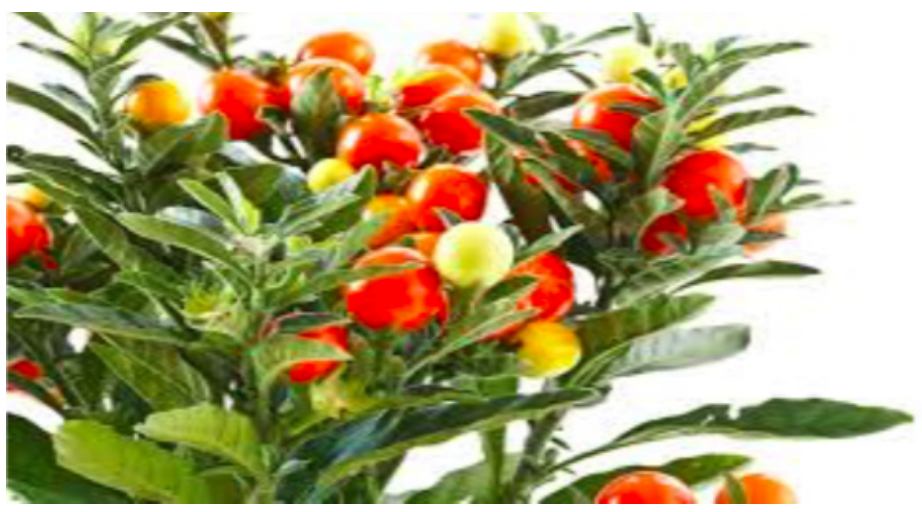

Figure 1: Image of the Ashwagandha plant (Withania somnifera) with its typical smallish green leaves and red and yellow berries. Public domain.

Though the leaves and berries have also been tested and used for medicinal properties, Ashwagandha's main therapeutic components are found in its whitish-brown roots that can grow up to 2 feet in length Withania Somnifera, 2004 (Figure 2).

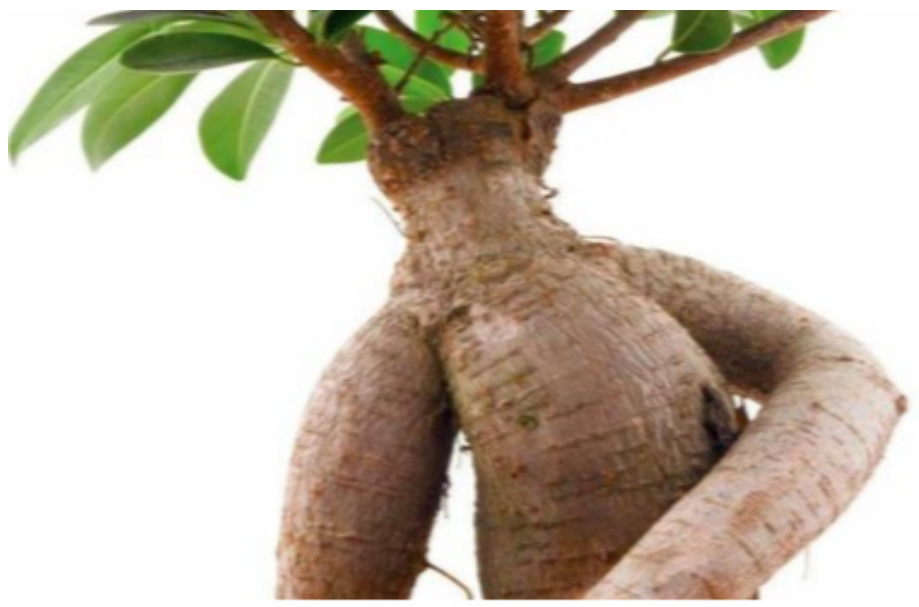

Figure 2: Image of the Ashwagandha plant (Withania somnifera) with its native whitish-brown roots. Public domain.

The plant commonly grows in India, especially in areas closest to the Himalayan region. It has been found growing in Africa and the Mediterranean. It blooms year-round and can survive in hot, arid conditions where many other plants cannot. In Ayurveda, plants that can survive in harsh conditions are considered to have strong healing and tonification properties Withania Somnifera, 2004.

Ashwagandha is a known adaptogen; therefore, each TCM herb that was used for comparison is considered apoptogenic. A comparison of the active chemical constituents was made, including phytochemicals and alkaloids, to demonstrate a distinctly close relationship-on a chemical level-among the herbs compared.

According to the online dictionary meriam-webster.com, an adaptogen or apoptogenic substance is "a nontoxic substance, and especially a plant extract, that is held to increase the body's ability to resist the damaging effects of stress and promote or restore normal physiological"; in other words, an herb used to restore homeostasis. To continue from the website medical-dictionary.thefreedictionary.com: "adaptogens may act on serum glucose, leukocytes, temperature, blood pressure or pulse by increasing or decreasing the substance of interest" (Table 2) 


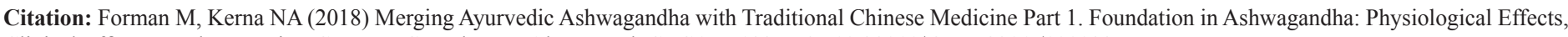
Clinical Efficacy, and Properties. Curr Res Complement Altern Med. CRCAM-133. DOI:10.29011/ 2577-2201 /100033

\begin{tabular}{|l|l|l|}
\hline \multicolumn{1}{|c|}{ Discipline } & \multicolumn{1}{|c|}{ Classification } & \multicolumn{1}{|c|}{ Actions } \\
\hline Ayurvedic practitioners & Rasayana & Rejuvenates \\
\hline TCM Doctors & Qi tonic & $\begin{array}{l}\text { Superior herb; Strengthens the } \\
\text { defense functions; Tonifies } \\
\text { defensive Qi }\end{array}$ \\
\hline Russian scientists & Adaptogen & $\begin{array}{l}\text { Increases the resistance within the } \\
\text { body to a wide range of stressors } \\
\text { and normalizes functions }\end{array}$ \\
\hline Western scientists & Adaptogen & $\begin{array}{l}\text { Regulates the HPA axis and } \\
\text { sympathetic responses }\end{array}$ \\
\hline Clinical herbalists & Adaptogen & $\begin{array}{l}\text { Re-regulates disharmonies in the } \\
\text { neuroendocrine and immune } \\
\text { systems }\end{array}$ \\
\hline
\end{tabular}

Table 2: A Comparison of Adaptogens in Different Medical Systems.

Note: Comparison of classification and actions of adaptogens among various disciplines. Adapted from D. Winston and S. Maimes [5].

\section{Preparation}

Ashwagandha is primarily prepared and used as a fine powder made from drying and crushing the roots. It has, however, been prepared in a variety of ways. In Ayurveda, the preparation of many herbs corresponds to the effect one is trying to achieve with that herb [6].

The most common traditional preparation of Rasayanas is called the "milk decoction". The usual method is to mix one-part herb with eight parts of milk and thirty-two parts of water and boil it with low heat until the water is evaporated.

In the case of Ashwagandha, however, the preferred method is to cook the herb directly with milk. It is said that milk augments the tonifying and nutritive effects of the herb. It is important to note that the preparation should take place in an earthenware pot; it is also said that earthenware combines with the herbs in a similar way plants combine with the soil. Currently, Ashwagandha is most often taken in the form of a powder extract of high concentration, usually in capsules. [6].

\section{Ashwagandha's Active Constituents}

Various studies have sought to discover the constituents responsible for Ashwagandha's observed and professed medicinal properties. Alkaloids are the primary chemical constituents of the plant including Isopelletierine, Anaferine, Cuseohygrine, Anahygrine, Somniferine, Somnine, Somniferinine, Withananine, Pseudo-Withanine, Tropine, Pseudo-Tropine, and Cuscohygrine [7]. It also contains significant amounts of saponins and steroidal lactones including withanolides and withaferins [8-10].
Currently, twelve alkaloids including thirty-five withanolides and many sitoindosides (withanolides containing glucose at C-27) have been isolated from the plant and studied. The withanolides have been extensively investigated and are believed to be the main constituents responsible for Ashwagandha's medicinal effects. Two withanolides, withaferin A and withaferin D, were found to be the most medicinally active [11]. Withanolides are steroidal and resemble the ginsenosides found in Panax ginseng (Ginseng) both in structure and activity [4].

\section{Alkaloids, Withanolides, and Sitoindosides (Glycowithanolides)}

Alkaloids are active compounds, found in naturally occurring substances, consisting of nitrogen-containing bases. There are many well-known alkaloids, such as morphine, strychnine, quinine, ephedra, and nicotine. They are found in flowering varieties of plants and have been studied extensively. Alkaloids are believed to be the waste materials given off by a plant during the metabolic process and are very powerful. It has been suggested that they may play a role in a natural insecticide action as a mechanism to preserve plant quality [12]. The primary chemical structures of alkaloids contain at least one nitrogen atom in an amine type structure. They are called alkaloids because, like their inorganic counterparts, they react with acids to form salts [12].

The medical properties of alkaloids are well known; they are potent and have been the basis for the formation of many, if not most, pharmaceutical drugs over the past 100 years. Many analgesics, like morphine and codeine, are derived from plants, and less addictive substances have been developed that treat cardiac and respiratory impairments. One of the more common alkaloids is nicotine derived from the tobacco plant and smoked in cigarettes, cigars, and pipes. Many hallucinogens are alkaloidbased substances, such as mescaline and psilocybin mushrooms; these have given way to inorganic hallucinogens like LSD made from lysergic acid [12].

Withanolides, a type of alkaloid found in Ashwagandha, are believed to be the active ingredient that provides the healing effects. The primary withanolides, which have been isolated chemically, are withaferin A and withaferin D. These two isolates are steroidal; they are fat-soluble organic compounds that synthesize readily in the body and can function as signaling molecules to affect the cell membranes. These characteristics help explain many of the anti-inflammatory and analgesic properties associated with Ashwagandha, like those found in other alkaloid plant compounds [13]. Sitoindosides (Glucowithanolides) are withanolides containing a glucose molecule at carbon-27. The active sitoindosides are sitoindoside VII and VIII; they have been shown to have a dramatic inhibitory effect on the inflammatory process [9]. 


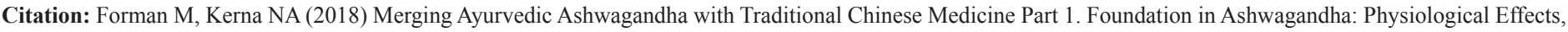
Clinical Efficacy, and Properties. Curr Res Complement Altern Med. CRCAM-133. DOI:10.29011/ 2577-2201 /100033

\section{Doses and Contraindications}

Ashwagandha has been recognized for thousands of years as an herb that can be taken long term with no toxic effects. It is extremely safe when used in a predefined range of doses. Many toxicity studies have indicated Ashwagandha to be completely safe [14]. A study by Sharma et al., as cited in Aphale, Chhibba, Kumohakarna, Mateebuddin, \& Dahat (1998) [15], showed that Ashwagandha exhibited no toxic effects in laboratory rats after eight months of continuous daily dosing. A follow-up study by Aphale et al. (1998) [15] also showed no significant organ damage from prolonged doses of Ashwagandha.

A study by Raut et al. (2012) [16] on the human tolerability of Withania (in which 18 subjects were given doses of aqueous extract of Ashwagandha root in doses of $750 \mathrm{mg} /$ day graduating to $1,250 \mathrm{mg}$ /day for 30 days) found no immediate intolerance or adverse effects in any vital functions or organ health. Also, no adverse effects were noted in the quality of sleep, appetite or bowel habits. Padmavathi, Rath, Rao, \& Singh (2005) [17] showed that continuous doses of Withania had no adverse effects on the P450 system in laboratory mice and body weight gain profile.

The recommended dosage of the roots is different for each form of the herb and different depending on the source. Yance (2013) [14] suggests-for an average size adult-the raw dried root can be taken in doses of 3-10 grams per day, a standardized powder extract (1:1) up to $1000 \mathrm{mg} /$ day or a concentrated liquid extract 2-8 $\mathrm{ml}$ per day. Other researchers suggest slightly higher doses; with all agreeing that staying within recommended ranges is critical for long-term safety. As with any substance, large doses should be avoided and have been associated with gastrointestinal upset, diarrhea, and vomiting. Large quantities may also be abortifacient and should, therefore, be used with caution during pregnancy. [14]

\section{Conclusion}

Traditional Chinese Medicine has a 2,000-year recorded history. Most of TCM's historical findings, prescriptions, and practices are noted in the extensive Materia Medica which was compiled over twenty-seven years during the Ming Dynasty by a scientific naturalist, Li Shizhen; the first draft was completed in $1578 \mathrm{AD}$. At that time, due to the long distances and lack of prompt communications between other countries and cultures, many herbs, renowned in their country of origin-such as Ashwagandha from India's Ayurvedic tradition-were not known or included in these earlier Chinese reference books. Even though Ashwagandha has been proven over Ayurvedic's 6,000-year history, it is yet to be included in TCM's Materia Medica. Currently, studies are underway to investigate Ashwagandha's bioactive physiological components on a microbiological level that seem to support certain practitioners' and patients' historical health benefit claims.
Ashwagandha is scientifically classified as Withania somnifera (Dunal); it belongs to the botanical family Solanaceae. It is a sturdy plant, of two to five feet in height, able to survive in hot climates. Ayurvedic considers such plants to have strong healing and tonification properties. Its whitish-brown roots are rich in bioactive ingredients of alkaloids, withanolides, and sitoindosides (glycowithanolides); therefore, it is usually ingested in the form of a powder in a liquid mix or capsule form. If consumed in the recommended doses, Ashwagandha can be taken long term without any significant adverse effects. Caution must be used in pregnancy as Ashwagandha has potential abortifacient properties. In a sideby-side comparison of Ayurvedic and TCM herbs, Ashwagandha can be categorized in TCM as having the properties to "Tonify Qi" and "Tonify Blood and Essence".

In subsequent articles, Ashwagandha will be investigated as an adaptogenic herb. Also, the physiological effects of Ashwagandha will be explored including anti-inflammatory and analgesic properties, immunostimulating and immunoprotective properties, neuroprotective and neuroregenerative properties, anticancer and anti-tumor properties, among others. A review of TCM and Ayurvedic herb classifications will follow, as well as a specific and comparative study between Ashwagandha and five fundamental TCM herbs. The series will conclude with a summary and support for Ashwagandha being included in Materia Medica resulting in more specific and effective treatment by Traditional Chinese Medicine practitioners, in being readily available and confidently utilized by the public, and in gaining further recognition, acceptance, and integration by Western allopathic medicine.

\section{Acknowledgments}

None

\section{Conflict of Interest Statement}

The authors declare that this paper was written in the absence of any commercial or financial relationships that could be construed as a potential conflict of interest.

\section{References}

1. Chen J, Chen T (2009) Chinese Medical Herbology and Pharmacology. City of Industry, California: Art of Medicine Press

2. Bhattacharya SK, Satyan KS, Ghosal S (1997) Antioxidant activity of glycowithanolides from Withania somnifera. Indian Journal of Experimental Biology 35: 236-239.

3. Chandrasekhar K, Kapoor J, Anishetty S (2012) A prospective, randomized double-blind, placebo-controlled study of safety and efficacy of a high-concentration full-spectrum extract of Ashwagandha root in reducing stress and anxiety in adults. Indian J Psychol Med 34: 255.

4. Singh N, Bhalla M, De Jager P, Gilca M (2011) An Overview on Ashwagandha: A Rasayana (Rejuvenator) of Ayurveda. African Journal of 


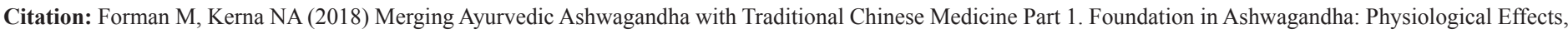
Clinical Efficacy, and Properties. Curr Res Complement Altern Med. CRCAM-133. DOI:10.29011/ 2577-2201 /100033

Traditional, Complementary and Alternative Medicines, 8: 208-213.

5. Winston D, Maimes S (2007) Adaptogens Herbs for Strength, Stamina and Stress Relief. Rochester, Vermont: Healing Arts Press 2007.

6. Frawley D, Lad V (2001) The Yoga of Herbs. Twin Lakes, Wisconsin: Lotus Press 2001.

7. Singh G, Sharma P, Dudhe R, Singh S (2010) Biological activities of Withania somnifera. Annals of Biological Research 1: 56-63.

8. Ven Murthy MR, Ranjekar PK, Ramassamy C, Deshpande M (2010) Scientific basis for the use of Indian ayurvedic medicinal plants in the treatment of neurodegenerative disorders: ashwagandha. Cent Nerv Syst Agents Med Chem. 2010 10: 238-246.

9. Mishra L, Singh B, Dagenais S (2000) Scientific Basis for the Therapeutic Use of Withania somnifera (Ashwagandha): A Review. Alternative Medicine Review 5: 334-346.

10. Nagappan A, Karunanithi N, Sentrayaperumal S, Park K, Park H, et al. (2004) Monograph. Angelica sinensis. Alternative Medicine Review 9: 429-433.

11. Shin H, Jeong H, An H, Hong S, Um J, et al. (2006) The effect of Panax Ginseng on forced immobility time and immune function in mice. Indian Journal of Medical Research 124: 199-206.

12. Encyclopedia Britannica (2015) A. Alkaloid.
13. Ichikawa H, Takada Y, Shishodia S, Jayaprakasam B, Nair MG, et al. (2006) Withanolides potentiate apoptosis, inhibit invasion, and abolish osteoclastogenesis through suppression of nuclear factor-KB (NF-KB) activation and NF-KB- regulated gene expression. Mol Cancer Ther 5:1434-1445

14. Yance D (2013) Adaptogens in Medical Herbalism. Rochester, VT: Healing Arts Press.

15. Aphale AA, Chhibba AD, Kumohakarna NR, Mateenuddin M, Dahat SH (1998) Subacute Toxicity Study of the Combination of Ginseng (Panax Ginseng) and Ashwagandha (Withenia Somnifera) in Rats: A Safety Assesment. Indian Journal of Physiol Pharmacol 42: 299-302.

16. Raut AA, Rege NN, Tadvi FM, Solanki PV, Kene KR, et al. (2012) Exploratory study to evaluate tolerability, safety, and activity of Ashwagandha (Withania somnifera) in healthy volunteers. Journal of Ayurveda and Integrative Medicine 3: 111-114.

17. Padmavathi B, Rath PC, Rao AR, Singh RP (2005) Roots of Withania somnifera Inhibit Forestomach and Skin Carcinogenesis in Mice. Evidence-based Complementary and Alternative Medicine 2: 99-105. 